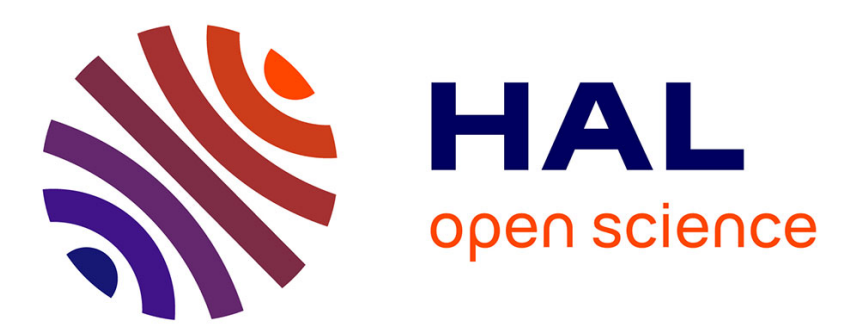

\title{
Investigation of the validity of Dynamic AcoustoElastic Testing for measuring nonlinear elasticity
}

\author{
M. Scalerandi, S. Gliozzi, S. Haupert, G. Renaud, M. Ait Ouarabi, F.
} Boubenider

\section{- To cite this version:}

M. Scalerandi, S. Gliozzi, S. Haupert, G. Renaud, M. Ait Ouarabi, et al.. Investigation of the validity of Dynamic AcoustoElastic Testing for measuring nonlinear elasticity. Journal of Applied Physics, 2015, 118 (12), pp.124905. 10.1063/1.4931917 . hal-01397214

\section{HAL Id: hal-01397214 \\ https://hal.sorbonne-universite.fr/hal-01397214}

Submitted on 15 Nov 2016

HAL is a multi-disciplinary open access archive for the deposit and dissemination of scientific research documents, whether they are published or not. The documents may come from teaching and research institutions in France or abroad, or from public or private research centers.
L'archive ouverte pluridisciplinaire HAL, est destinée au dépôt et à la diffusion de documents scientifiques de niveau recherche, publiés ou non, émanant des établissements d'enseignement et de recherche français ou étrangers, des laboratoires publics ou privés. 


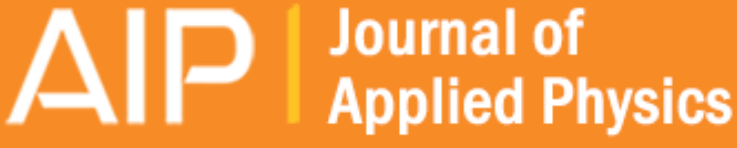

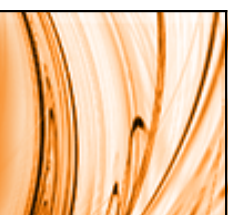

\section{Investigation of the validity of Dynamic AcoustoElastic Testing for measuring nonlinear}

elasticity

M. Scalerandi, A. S. Gliozzi, S. Haupert, G. Renaud, M. Ait Ouarabi, and F. Boubenider

Citation: Journal of Applied Physics 118, 124905 (2015); doi: 10.1063/1.4931917

View online: http://dx.doi.org/10.1063/1.4931917

View Table of Contents: http://scitation.aip.org/content/aip/journal/jap/118/12?ver=pdfcov

Published by the AIP Publishing

\section{Articles you may be interested in}

Modeling dynamic acousto-elastic testing experiments: Validation and perspectives

J. Acoust. Soc. Am. 136, 1530 (2014); 10.1121/1.4893907

Publisher's Note: "Pump and probe waves in dynamic acousto-elasticity: Comprehensive description and comparison with nonlinear elastic theories" [J. Appl. Phys. 114, 054905 (2013)]

J. Appl. Phys. 114, 089903 (2013); 10.1063/1.4820251

Pump and probe waves in dynamic acousto-elasticity: Comprehensive description and comparison with nonlinear elastic theories

J. Appl. Phys. 114, 054905 (2013); 10.1063/1.4816395

Anisotropy of dynamic acoustoelasticity in limestone, influence of conditioning, and comparison with nonlinear resonance spectroscopy

J. Acoust. Soc. Am. 133, 3706 (2013); 10.1121/1.4802909

Remote dynamic acoustoelastic testing: Elastic and dissipative acoustic nonlinearities measured under hydrostatic tension and compression

Appl. Phys. Lett. 94, 011905 (2009); 10.1063/1.3064137

\section{AIP $\mid$ APL Photonics}

APL Photonics is pleased to announce Benjamin Eggleton as its Editor-in-Chief

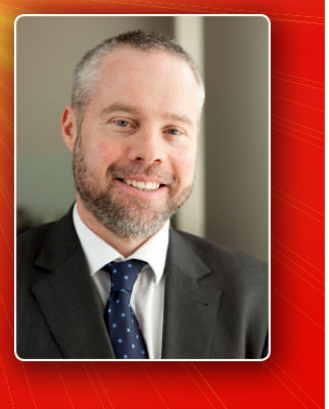




\title{
Investigation of the validity of Dynamic AcoustoElastic Testing for measuring nonlinear elasticity
}

\author{
M. Scalerandi, ${ }^{1}$ A. S. Gliozzi, ${ }^{1}$ S. Haupert, ${ }^{2,3,4}$ G. Renaud, ${ }^{2,3,4}$ M. Ait Ouarabi, ${ }^{5}$ \\ and F. Boubenider ${ }^{5}$ \\ ${ }^{1}$ Department of Applied Science and Technology, Politecnico di Torino, Corso Duca degli Abruzzi 24, \\ 10129 Torino, Italy \\ ${ }^{2}$ Sorbonne Universités, UPMC Univ Paris 06, UMR 7371, UMR_S 1146, Laboratoire d'Imagerie Biomédicale, \\ F-75006, Paris, France \\ ${ }^{3}$ CNRS, UMR 7371, Laboratoire d'Imagerie Biomédicale, F-75006, Paris, France \\ ${ }^{4}$ INSERM, UMR_S 1146, Laboratoire d'Imagerie Biomédicale, F-75006, Paris, France \\ ${ }^{5}$ Laboratoire de Physique des Matèriaux, Universitè des Sciences et de la Technologie Houari Boumediene, \\ BP 32 El Alia, 16111, Bab Ezzouar, Alger, Algeria
}

(Received 6 May 2015; accepted 16 September 2015; published online 30 September 2015)

\begin{abstract}
Measurement of the dependence of the elastic moduli on the strain, i.e., the characterization of nonlinear elastic properties of solid media, poses intrinsic experimental difficulties. The Dynamic AcoustoElastic Technique has been recently developed as an efficient tool for the determination of the modulus in both compression and tension. The goal of the present paper is to discuss the limitations of the experimental implementation and the interpretation of the measured quantities in terms of nonlinear parameters. For this purpose, simulation results will be presented for both classical and nonclassical nonlinear elastic media. @ 2015 AIP Publishing LLC. [http://dx.doi.org/10.1063/1.4931917]
\end{abstract}

\section{INTRODUCTION}

In order to understand the physical mechanisms responsible for elastic nonlinearity in solid media, the knowledge of the dependence of the elastic modulus on strain is needed, together with the monitoring of its variation when varying physical properties such as humidity, ${ }^{1}$ temperature, ${ }^{2}$ and applied stress. ${ }^{3}$ This is particularly true in the case of nonclassical nonlinear elastic media, ${ }^{4}$ e.g., in the case of rocks, ${ }^{5}$ concrete, ${ }^{6}$ damaged metals, ${ }^{7,8}$ bones, ${ }^{9}$ and composites. ${ }^{10}$ In these materials, in fact, hysteretic loops in the stress strain relation, ${ }^{11}$ conditioning, ${ }^{12}$ and relaxation ${ }^{13}$ effects make the understanding of the dynamic behavior of the system studied highly complex.

In general, the nonlinearity of a material is quantified introducing the so called nonlinear elastic tensors $\beta$ and $\delta$. Normally, scalar stress-strain constitutive equations are formulated. Within these formulations, in the case of classical nonlinearity, stress $\sigma$ is assumed to be cubically dependent on strain $\epsilon: \sigma=S\left(\epsilon+\beta \epsilon^{2}+\delta \epsilon^{3}\right) .{ }^{14}$ Here, $S$ is the linear modulus. In this paper, we will discuss the limitations of such scalar formulation and the implications about the physical interpretation of the results of measurements of the modulus.

In the case of nonclassical media, both tensors are themselves strain dependent, as it is evident from the deviation from the parabolic behavior of the curve of the modulus vs. $\epsilon{ }^{11}$ In practice, the problem is generally simplified, with good accuracy, assuming different nonlinear parameters for the upgoing (increasing strain) and downgoing (decreasing strain) branches of the $\sigma$ vs. $\epsilon$ curves. ${ }^{15}$

The measurement of the modulus as a function of strain, i.e., the determination of the nonlinear parameters, poses intrinsic difficulties. Quasistatic experiments measure directly the dependence of the stress on strain. ${ }^{16}$ These measurements are difficult to perform, particularly in tension and at low strain levels (smaller than $10^{-5}$ ).
As an alternative, in the last decades, Static AcoustoElastic Testing (SAET) has been proposed. ${ }^{17-21}$ In this approach, a quasistatic loading is applied and the modulus tested at the given pressure by measuring the speed of sound of a probing pulse. While a high precision in the determination of the nonlinear parameters is obtained, it is again difficult to implement SAET experiments, particularly with a tensile strain and at low strain levels.

To allow measurements in both tension and compression at low strain levels, a new technique has emerged in the last decade, named Dynamic AcoustoElastic Testing (DAET). ${ }^{22-27}$ The method is very similar to a SAET approach, except that the loading is induced by a low frequency (LF) sinusoidal excitation perturbing the material in standing wave conditions. If the time-of-flight (TOF) to traverse the sample is very short, the probing pulse feels an almost constant confining pressure along its propagation path. The method allows one to monitor both tensile and compressive phases and to excite, at least in principle, arbitrarily small confining strains in the material. ${ }^{22}$

Some issues related to the practical implementation of DAET are, however, still not completely discussed in the literature and their study is the goal of the present paper. First, in a DAET experiment, normally only one strain component is measured and the modulus is shown as a function of this strain. As a consequence, a scalar value of the nonlinear parameters $\bar{\beta}$ and $\bar{\delta}$ is in general given. ${ }^{22,28-31}$ These scalar values are therefore an estimate of a complex function of the components of the tensorial nonlinear parameters.

The second issue is mostly linked to the experimental implementation, and it is discussed in the Supplementary material, ${ }^{32}$ where the assumptions linked to the choice of the pulse frequency and pump amplitude are discussed. As we will show, normally the configuration adopted is not significantly influencing results. 
To analyze the two issues, a numerical analysis is performed. We will use two distinct models for classical and nonclassical nonlinearities, discussed in Section II. The models presented are a modification of the models already presented in Ref. 33, where non tensorial nonlinear effects (classical and nonclassical) were considered. In Section III, simulations of both DAET and SAET experiments will be performed and compared to validate the DAET approach. Simulation results will be compared with quasistatic predictions, and we will discuss the influence on the measurements due to the nonlinear dependence of the Poisson ratio $\nu$ and of the attenuation coefficient.

\section{THEORY}

\section{A. Classical nonlinear elasticity model}

\section{Equation of state}

We consider a 2-D system in the general case of an anisotropic material assuming in-plane stress/strain components. Thus, all partial derivatives in the out-of-plane direction are vanishing and the corresponding components of the modulus are not relevant in the wave equation. Therefore, assuming orthotropic symmetry, we have to consider only four independent elastic constants that we note: $S_{1}=S_{1111}, S_{2}=S_{2222}, S_{3}=S_{1122}$, and $S_{4}=S_{1212}$ and permutations. In the following, the indexes 1 and 2 denote the $x$ and $y$ directions, respectively. The equation of state, linking the stress $(\sigma)$ components to the strain $(\epsilon)$ components, is

$$
\begin{aligned}
& \sigma_{11}=S_{1} \epsilon_{11}+S_{3} \epsilon_{22}, \\
& \sigma_{22}=S_{3} \epsilon_{11}+S_{2} \epsilon_{22}, \\
& \sigma_{12}=S_{4} \epsilon_{12},
\end{aligned}
$$

where

$$
\begin{aligned}
S_{j}= & S_{j}^{l i n}\left(1+\beta_{j 1} \epsilon_{11}+\beta_{j 2} \epsilon_{22}+\beta_{j 3} \epsilon_{12}+\delta_{j 1} \epsilon_{11}^{2}+\delta_{j 2} \epsilon_{22}^{2}\right. \\
& \left.+\delta_{j 3} \epsilon_{12}^{2}\right) .
\end{aligned}
$$

Note that additional quadratic terms involving the cross product of different strain components could also be added in Eq. (2). Even though they might give contributions of the same order of magnitudes as the terms considered, including them explicitly in the formulation is not providing additional support to the claim that a physical interpretation of DAET results is possible only when considering proper combinations of nonlinear elastic constants. Thus, to limit the complexity in the mathematical formulation of the problem, we have omitted them.

\section{Equations of motion}

In the case of a 2-D system, we discretise the sample as a set of $N_{1} \times N_{2}$ square elements of size $h$. Each node of the grid, with discrete coordinates $(i, j)$, corresponds to a cell representing a portion of the material of density $\rho$. A simplified approach, with a scalar description of the nonlinear elastic modulus, was already introduced in Ref. 33.
Denoting with $u$ and $v$ the components of the displacement vector of the node $(i, j)$, the following equations of motion are valid:

$$
\begin{aligned}
\rho \ddot{u}-\gamma \dot{u} & =\partial_{l} \sigma_{1 l}=\partial_{l}\left(S_{1 l m n} \epsilon_{m n}\right), \\
\rho \ddot{v}-\gamma \dot{v} & =\partial_{l} \sigma_{2 l}=\partial_{l}\left(S_{2 l m n} \epsilon_{m n}\right),
\end{aligned}
$$

where $\sigma_{k l}$ and $\epsilon_{m n}$ are the stress and strain components and $k$, $l=1,2$. The first order time derivative term accounts for viscous attenuation with a dynamic viscosity $\gamma$, which is assumed as simple as possible (i.e., without any tensorial dependence) to simplify the discussion. Repeated indexes imply summation.

If we discretise Eq. (3), using a standard Finite Difference forward scheme, we obtain an equivalent formulation ${ }^{34}$ assuming the node $i, j$ as subject to a combination of volumetric forces $\vec{F}_{k}$ (forces per unit volume), imposed by the four adjacent cells (see Fig. 1)

$$
\rho\left(\begin{array}{c}
\ddot{u} \\
\ddot{v}
\end{array}\right)-\gamma\left(\begin{array}{c}
\dot{u} \\
\dot{v}
\end{array}\right)=\sum_{k=1}^{8} \vec{F}_{k} .
$$

The 8 applied terms $\vec{F}_{k}$, given by the ratio between stresses and lattice size, can be derived as

$$
\vec{F}_{k}=M_{k} \Delta w_{k},
$$

where

$$
\begin{aligned}
& M_{k}=\left(\begin{array}{cc}
0 & \frac{1}{4}\left(S_{3}+S_{4}\right) \\
\frac{1}{4}\left(S_{3}+S_{4}\right) & 0
\end{array}\right) \quad(k=1,2,3,4), \\
& M_{k}=\left(\begin{array}{cc}
\frac{1}{2} S_{1} & -\frac{1}{4}\left(S_{3}-S_{4}\right) \\
\frac{1}{4}\left(S_{3}-S_{4}\right) & \frac{1}{2} S_{4}
\end{array}\right) \quad(k=5,7), \\
& M_{k}=\left(\begin{array}{cc}
\frac{1}{2} S_{4} & \frac{1}{4}\left(S_{3}-S_{4}\right) \\
-\frac{1}{4}\left(S_{3}-S_{4}\right) & \frac{1}{2} S_{2}
\end{array}\right) \quad(k=6,8),
\end{aligned}
$$

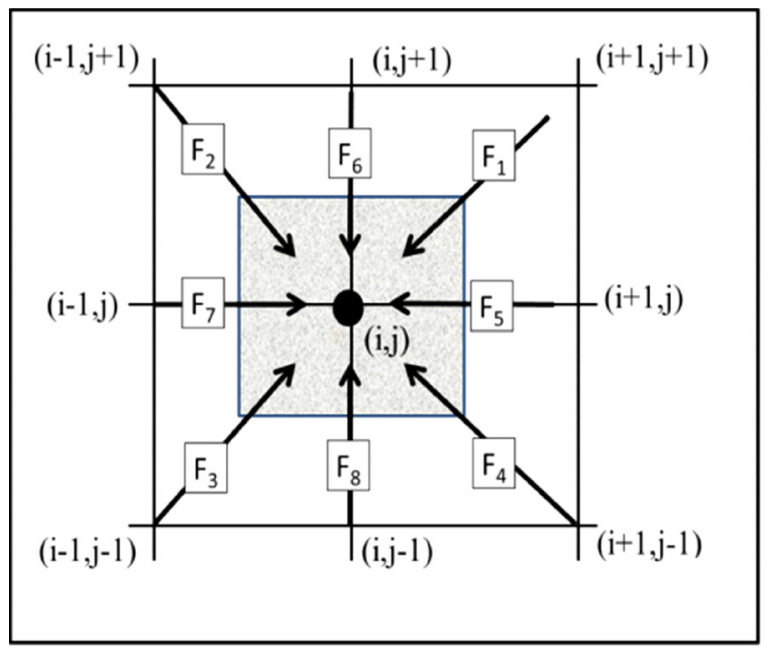

FIG. 1. Discretization scheme and forces acting on a given node of the grid 
and

$$
\Delta w_{k}=\frac{1}{h}\left(\begin{array}{c}
u_{k}-u \\
v_{k}-v
\end{array}\right),
$$

where $u_{k}$ and $v_{k}$ are the components of the displacement vector of the node in the direction $k$ with respect to $(i, j)$, and we recall that $h$ is the lattice step. For example, in the case $k=5$, it is the displacement vector of the node $(i+1, j)$. In the used notation, $\Delta w_{k}$ is a strain tensor.

It follows

$$
\begin{aligned}
& \rho \ddot{u}-\gamma \dot{u}=\sum_{k=1}^{8}\left(f_{k}^{(1,1)}+f_{k}^{(1,2)}\right), \\
& \rho \ddot{v}-\gamma \dot{v}=\sum_{k=1}^{8}\left(f_{k}^{(2,1)}+f_{k}^{(2,2)}\right),
\end{aligned}
$$

where

$$
\begin{aligned}
f_{k}^{(1,1)} & =M_{k}(1,1) \frac{u_{k}-u}{h} \quad f_{k}^{(1,2)}=M_{k}(1,2) \frac{v_{k}-v}{h}, \\
f_{k}^{(2,1)} & =M_{k}(2,1) \frac{u_{k}-u}{h} \quad f_{k}^{(2,2)}=M_{k}(2,2) \frac{v_{k}-v}{h} .
\end{aligned}
$$

The components of the matrices $M$ are the moduli (see Eq. (7)), which are defined by the material equation of state given by Eq. (2). The strain components in the discrete scheme are given as

$$
\begin{aligned}
\epsilon_{11}= & 0.5\left(u_{i+1, j+1}+u_{i+1, j-1}-u_{i-1, j+1}-u_{i-1, j-1}\right) /(2 h) \\
\epsilon_{22}= & 0.5\left(v_{i+1, j+1}+v_{i-1, j+1}-v_{i+1, j-1}-v_{i-1, j-1}\right) /(2 h) \\
\epsilon_{12}= & 0.25\left(u_{i+1, j+1}-u_{i+1, j-1}+u_{i-1, j+1}-u_{i-1, j-1}+\right. \\
& \left.+v_{i+1, j+1}-v_{i-1, j+1}+v_{i+1, j-1}-v_{i-1, j-1}\right) /(2 h) .
\end{aligned}
$$

\section{B. Hysteretic model}

\section{Equation of state}

Several models have been proposed in the literature to describe a hysteretic behavior in the quasistatic equation of state. $^{35-37}$ Here, we used a model very similar to that proposed in Refs. 12 and 38, which is adequate to describe the behavior of rocks and concrete. In other cases, such as when dealing with bones, the hierarchical structure of the medium might require different approaches. A simplified similar model was also used in Ref. 33 .

The model is based on a Preisach-Mayergoyz space approach, in which the macroscopic stress-strain relation is derived by a proper statistical averaging over a large number of microscopic elements, called Hysteretic Elements (HE). In the formulation introduced in Subsection II A, the motion of each grid points is governed by several springs, which generate forces in the form $f=S \epsilon$ (see Eq. (9)). The hysteretic behavior of the material is thus simulated replacing each of these springs with a "scalar" bi-state spring described heuristically as follows.

We consider as the basic Hysteretic Element a spring that may be in one of the two states, rigid or elastic. Note that here we assume a purely elastic state, even though classical nonlinear terms linked explicitly to $\beta$ and $\delta$ could also be eventually considered. However, the model proposed accounts for both hysteresis loops and curvature in the stress-strain dependence. The modulus of the spring is

$$
\tilde{K}= \begin{cases}k_{1}=K & \text { state } 1 \\ k_{2}=\infty & \text { state } 2 .\end{cases}
$$

The spring resides in one of the two states depending on the value of a control variable $\Lambda$, which could be any component of the strain tensor $\epsilon$ and switches from one state to the other when the control variable varies. In particular (see Fig. 2(a)), if the spring is in state 1 when $\Lambda<\Lambda_{o}$, when $\Lambda$ increases above a threshold value $\Lambda_{c}$, the spring switches to the rigid state. When $\Lambda$ decreases below $\Lambda_{o}$, the spring switches back to the state 1.

We then consider a set of springs arranged in series. For simplicity, but the generalization is straightforward, we consider all springs with the same elastic constant $K$ and the same length. On the contrary, to introduce a statistical ensemble, each spring is characterized by a different couple of values for the transition parameters $\left(\Lambda_{o}, \Lambda_{c}\right)$. It follows that, for a given value of the strain, the ensemble is characterized by a set of springs in the rigid and in the elastic state, which have the following deformations $\delta$ :
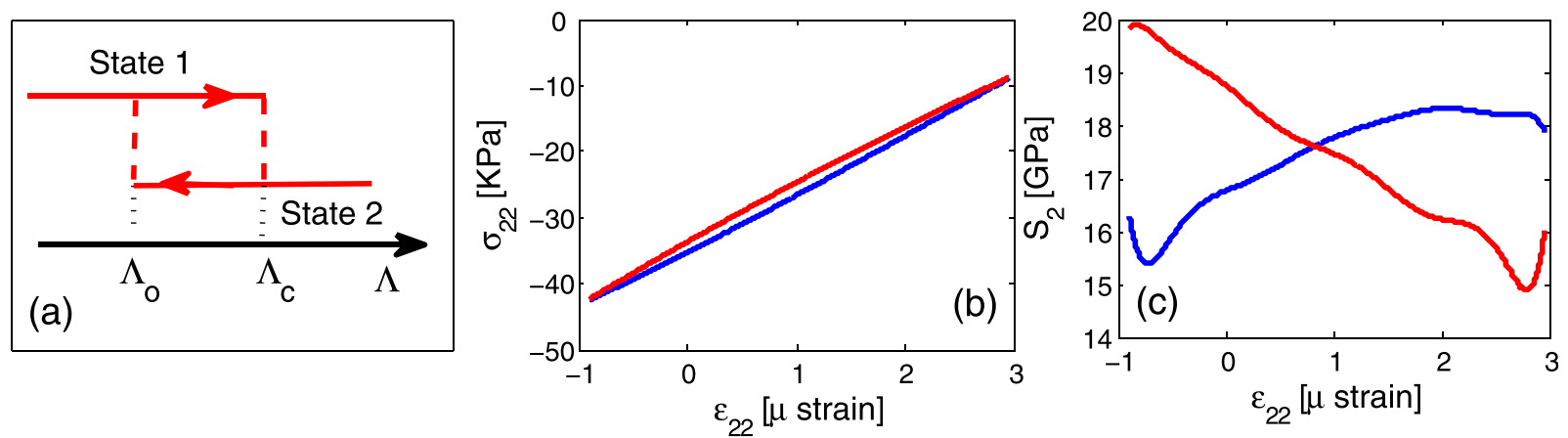

FIG. 2. Description of the nonlinear constitutive equations for hysteretic elasticity. (a) Description of the states of the hysteretic elements and the transition between states. (b) Example of stress-strain equation derived in the case of hysteretic elements depending only on $\epsilon_{22}$. (c) Modulus as a function of strain derived from subplot (b). 


$$
\begin{aligned}
\delta_{\text {elastic }} & =\sigma / K, \\
\delta_{\text {rigid }} & =\Lambda_{c} .
\end{aligned}
$$

Since the total strain is

$$
\epsilon=\sum_{\text {rigid }} \delta_{\text {rigid }}+\sum_{\text {elastic }} \delta_{\text {elastic }},
$$

we can derive

$$
\sigma=K / N_{\text {elastic }}\left[\epsilon-\sum_{\text {rigid }} \delta_{\text {rigid }}\right],
$$

where $N_{\text {elastic }}$ is the number of springs that, for the given value of strain, are in the elastic state.

In conclusions, to calculate the stress, it is sufficient to keep trace of the states of the springs when the strain varies. This could be achieved by following the protocol depicted in Fig. 2(a). An element can be activated by two or more strain components. To simulate a complex dependence, if we have $R$ springs, we consider four subsets, such that $R=\sum r_{i}$ :

- $r_{1}$ springs that are permanently in the elastic state, that is $\Lambda_{c}=\infty$;

- $r_{2}$ springs that have as a control variable $\epsilon_{11}$;

- $r_{3}$ springs that have as a control variable $\epsilon_{22}$;

- $r_{4}$ springs that have as a control variable $\epsilon_{12}$;

The major differences of the present model with respect to the model discussed in Ref. 33 are the choice of the control variable and the introduction of a tensorial behavior in the nonlinear response. Indeed, in the previous model, we have adopted the choice of the volumetric stress to be the control variable. This choice did not allow to describe any anisotropy in the nonlinear response, which is normally observed in quasistatic experiments for instance in rocks or materials with localized directional cracks.

In Fig. 2(b), we show a typical stress-strain relation derived from such implementation and the corresponding dependence of the modulus on $\epsilon_{22}$. For Fig. 2(b), we have assumed $R=100$ and $r_{1}=r_{3}=0.5 R, r_{2}=r_{4}=0$. The modulus has been derived as the partial derivative of $\sigma_{22}$ with respect to $\epsilon_{22}$. As already remarked, the hysteretic model proposed accounts for both curvature and loops in the stressstrain equation without including explicitly classical terms in the equation of state for the individual springs.

\section{Equations of motion}

The derivation of the equations of motion is conceptually simple, albeit mathematically complex. As mentioned before, the system is discretized in an $N_{1} \times N_{2}$ grid. Each cell is subject to 8 forces, which are a proper combination of the stresses, explicitly defined by Eq. (14). The same combination as for the classical case is still valid. At a given time step (in our approach time is also discretized), displacements for each grid point are known. The strain components are calculated for each cell and the state of the corresponding springs is updated. All strains could be derived using Eq. (14), and the corresponding forces derived and the displacements could be updated to the next time step using Eq. (4).
As mentioned, the degree of mathematical complexity is high. Indeed, in the most complex case, for each stress component (i.e., 5 components), the state of springs belonging to four classes should be updated. From the numerical point of view, this implies a significant memory occupation and a long computational time. The latter is in principle a problem, unless fast supercomputer is used. Alternative approaches to finite differences might help to make the code execution faster keeping the same/similar model description. ${ }^{39}$

\section{INTERPRETATION OF THE DAET PARAMETERS}

\section{A. Statement of the problem}

\section{Implementation of a DAET experiment}

In a DAET experiment, a sample is excited with a pump at LF wave and a probe at high frequency (HF). The pump is a longitudinal sinusoidal wave in standing wave conditions (the frequency is usually chosen as the first longitudinal resonance mode to optimize the energy of excitation). The probe is a short ultrasonic pulse, propagating in a direction orthogonal to the pump direction. Within each period of the pump, several impulse probes are sent into the sample. Each probe is thus traveling in a medium deformed differently by the pump. The TOF of the probe signal from the source to the receiver, normally located on opposite sides of the sample, is measured and could be plotted as a function of the pump strain.

The goal of a DAET experiment is to link the TOF to the modulus of the material and thus extract the dependence of the modulus from the strain and estimate the nonlinear coefficients $\beta$ and $\delta$ introduced above. Indeed, TOF is linked to the wave velocity $v_{L}$, which is linked to the modulus. Assuming the pulse propagating in the $y$-direction (dimension 2), we can define a modulus as

$$
\tilde{S}_{2}=v_{L}^{2} \rho(1+f(\gamma))=d^{2} / \operatorname{TOF}^{2} \rho(1+f(\gamma)),
$$

where $d$ is the distance between the HF source and the receiver. The term $f(\gamma)$ represents an unspecified contribution due to the attenuation, with a functional form depending on the kind of attenuation present in the medium. The wave velocity is related to the complex modulus, which in turn depends on the attenuation coefficient. As a result, the real part of the velocity is (weakly) dependent on $\gamma^{40}$ Normally, it is assumed $f(\gamma) \ll 1$ and the correction is neglected.

Finally, in a DAET experiment, the modulus is calculated for different values of the pump strain $\epsilon_{\text {pump }}$, and it is normally assumed to have a quadratic dependence ${ }^{29}$

$$
\tilde{S}_{2}=c_{D}+\beta_{D} \epsilon_{\text {pump }}+\delta_{D} \epsilon_{\text {pump }}^{2} .
$$

Notice that in experimental papers, usually the modulus variation (with respect to its linear value) is reported. In that case, the subscript $E$ is used for the modulus and $c_{E}=\left(c_{D}-S_{\text {lin }}\right) / S_{\text {lin }}$ and similar for $\beta$ and $\delta$.

The three parameters $c_{D}, \beta_{D}$, and $\delta_{D}$ are extracted fitting the curve of the variation of the time of flight or of the modulus as a function of the strain $\epsilon_{\text {pump }}$. If the case, as we will 
also discuss later, the upgoing and downgoing branches of the modulus vs. strain curve could be fitted independently.

\section{Link between DAET and quasistatic nonlinear parameters}

A first major issue in a DAET experiment is the definition of the strain in Eq. (16). The strain is a tensor, and thus several components might be independently influencing the modulus. In the following, we will limit the discussion to the classical nonlinear case, even though the conclusions are valid also for other cases, provided the modulus (at least independently for each branch) could be expanded as a quadratic polynomial. Additional terms in the expansion might be needed.

The modulus $\tilde{S}_{2}$ estimated from a DAET experiment should be equivalent to the modulus derived from the constitutive equation, which for the classical case are Eqs. (1) and (2)

$$
\begin{aligned}
\tilde{S}_{2}= & \frac{\partial \sigma_{22}}{\partial \epsilon_{22}}, \\
\tilde{S}_{2}= & S_{2}^{\operatorname{lin}}\left[1+\beta_{21} \epsilon_{11}+2 \beta_{22} \epsilon_{22}+\delta_{21} \epsilon_{11}^{2}+3 \delta_{22} \epsilon_{22}^{2}\right. \\
& \left.+\beta_{23} \epsilon_{12}+\delta_{23} \epsilon_{12}^{2}\right]+S_{3}^{l i n}\left[\beta_{32} \epsilon_{11}+2 \delta_{32} \epsilon_{22} \epsilon_{11}\right] .
\end{aligned}
$$

Notice that, in the geometry of a DAET experiment, a longitudinal stress is applied with symmetry in the y-direction. Thus, the pump strain is only in the directions 11 and 22 (due to the Poisson effect), while it is zero in the direction 12. Eventual effects due to nonclassical nonlinearities, which might introduce asymmetries, are in any case higher order effects with respect to those considered here. In the following, to simplify the notations, we will thus omit in all the relevant equations all terms proportional to $\epsilon_{12}$.

Equation (17) deserves a clear discussion. In nonlinear media, the only quantities with a physical interpretation are the elastic constants (linear and nonlinear) used in the right hand side of Eq. (2). The definition in Eq. (17) is only an empirical definition of the nonlinear modulus, which allows us to consider the material as locally linear elastic, i.e., linear elastic around given values of the strain components. However, as we will show numerically, this operational definition corresponds well to the variable measured in a DAET experiment. The independent reconstruction from Eq. (17) of all the physical parameters (components of the nonlinear elastic tensor) is an issue still to be addressed and should probably require independent DAET measurements.

Furthermore, we remark that the operational definition of the modulus measured in a DAET experiment (Eq. (17)) is not equivalent to the definition of Eq. (2). This becomes particularly relevant when considering nonclassical nonlinear media. Indeed, as remarked, the definition used here is equivalent to consider a linear elastic medium in a small interval around the strains induced by the pump. As a consequence, any dependence of the physical components of the nonlinear elastic tensor on higher order powers of the strain is simply hidden. In other words, when applying a quadratic fitting to measurements on nonclassical/hysteretic materials, the coefficients of the quadratic fitting of Eq. (16) are physically strain dependent themselves.

Equation (17) is too complex for practical purposes, since in experiments, only one component of the strain could in general be measured. To derive an equation similar to Eq. (16), we should therefore introduce some approximations, which will be numerically tested in Subsection III B. The first is to assume that the two strain components are linked through the Poisson ratio $\nu$, defined as the ratio between $S_{3}$ and $S_{2}$. In the general linear case, in quasistatic conditions and for a uni-axial test, we have

$$
\epsilon_{22}=-\nu \epsilon_{11} .
$$

This equation, valid only in the quasistatic limit, is applicable to the pump strain only, given the conditions imposed in a DAET experiment on the pump frequency, which is indeed equivalent to a uniaxial stress. However, since the pump strain is much larger than the probe strain, we could assume it to be approximately valid also when both pump and probe are propagating.

Assuming $\nu$ as strain independent, it follows:

$$
\begin{aligned}
\tilde{S}_{2}= & {\left[S_{2}^{l i n}+\left(S_{2}^{l i n} \beta_{21}-2 S_{2}^{l i n} \beta_{22} \nu+S_{3}^{l i n} \beta_{32}\right) \epsilon_{11}\right.} \\
& \left.+\left(S_{2}^{l i n} \delta_{21}+3 S_{2}^{l i n} \delta_{22} \nu^{2}-2 S_{3}^{l i n} \delta_{32} \nu\right) \epsilon_{11}^{2}\right],
\end{aligned}
$$

which allows for a comparison with the equation actually used in the analysis of experimental data.

As a consequence, the $\beta_{D}$ and $\delta_{D}$ parameters extracted from a DAET experiment through Eq. (16) are linked to the nonlinear parameters of the material by a complex relation

$$
\begin{aligned}
& \beta_{D}=S_{2}^{l i n} \beta_{21}-2 S_{2}^{l i n} \beta_{22} \nu+S_{3}^{l i n} \beta_{32}, \\
& \delta_{D}=S_{2}^{l i n} \delta_{21}+3 S_{2}^{l i n} \delta_{22} \nu^{2}-2 S_{3}^{l i n} \delta_{32} \nu .
\end{aligned}
$$

We remark that several assumptions introduced might render Eq. (20) only approximate

- we have assumed a linear Poisson ratio $\nu$;

- we have neglected the contribution from the attenuation term;

- the equations are valid only in the assumption of a pump strain that is constant during the propagation of the probe, which is not exactly true. Indeed, in experiments, normally the strain in Eq. (16) is calculated as the averaged strain during the propagation time. The correct equation should be

$$
\begin{gathered}
\tilde{S}_{2}=c_{D}+\beta_{D}\left\langle\epsilon_{\text {pump }}\right\rangle+\delta_{D}\left\langle\epsilon_{\text {pump }}^{2}\right\rangle, \\
\tilde{S}_{2} \approx c_{D}+\beta_{D}\left\langle\epsilon_{\text {pump }}\right\rangle+\delta_{D}\left\langle\epsilon_{\text {pump }}\right\rangle^{2} .
\end{gathered}
$$

In Subsection III B, all these issues will be discussed using numerical data to validate to which level the mentioned approximations are acceptable.

\section{Correction due to linear attenuation}

Even if we assume the nonlinear dependence of the attenuation coefficient to be negligible, the effects of linear attenuation should be considered, even though small. From 
Eq. (15), it is clear that attenuation (if strain independent) introduces a multiplicative constant (at the given frequency of the probe) in the definition of the wave velocity. This constant could be derived measuring the TOF in the absence of the pump wave and used to correct the results. In particular, in the absence of the pump

$$
k_{\text {att }}=v_{L}^{2} \rho / S_{2}^{\text {lin }}
$$

Therefore, when performing the analysis using DAET, we modify Eq. (15) as

$$
\tilde{S}_{2}=1 / k_{\text {att }} v_{L}^{2} \rho=1 / k_{\text {att }} d^{2} / T O F^{2} \rho .
$$

\section{B. Numerical results}

\section{Numerical experiment}

We have considered a $2 \mathrm{D}$ rectangular sample (elastically isotropic) with dimensions $10 \mathrm{~cm} \times 1 \mathrm{~cm}$, with linear elastic constants similar to those of rocks: $S_{1}=S_{2}=21 \mathrm{GPa}$ and $S_{4}=8 \mathrm{GPa}\left(S_{3}=S_{1}-2 S_{4}\right)$. The density is $\rho=2200 \mathrm{~kg} / \mathrm{m}^{3}$. The pump wave is generated from a transducer located on the surface of the sample $x=0$ and its frequency is $\omega_{L F}=7.537 \mathrm{kHz}$, corresponding to the first longitudinal resonance mode. Note that we have assumed forced-free boundary conditions; therefore, the first resonance mode corresponds to a wavelength that is a quarter of the sample size. The probe signal is a Gaussian modulated signal with frequency $\omega_{H F}=1 \mathrm{MHz}$ and duration $\Delta=4 \mu \mathrm{s} \approx T_{L F} / 33$, where $T_{L F}$ is the pump period. The probe transducer is located in $x=3.2 \mathrm{~cm} ; y=0$. The signal for the analysis is detected by a receiver in $x=3.2 \mathrm{~cm} ; y=1 \mathrm{~cm}$, unless otherwise specified.

The numerical experiment and data analysis are simulating exactly the procedure adopted in experiments. In all the cases considered here, the TOF is sufficiently small with respect to the LF period. Therefore, the assumption $\left\langle\epsilon^{2}\right\rangle \approx$ $\langle\epsilon\rangle^{2}$ is valid.

\section{Small classical nonlinearity}

The number of independent nonlinear elastic constants in the classical nonlinear model (Eq. (2)) is large. Since a DAET experiment is equivalent to a uni-axial test in the direction 1 , no shear strain is expected $\left(\epsilon_{12}=0\right)$. So, we can simplify Eq. (2) and keep only the nonlinear parameters attached to longitudinal strain. Also to reduce the number of model parameters, we assume $\beta_{11}=\beta_{22}=\beta_{1}, \beta_{12}=\beta_{21}=\beta_{2}$ and similar for $\delta$.

In the following, we will consider two simplified cases, in which only a few nonlinear coefficients are different from zero:

- CASE A: in the simplest case, we assume only one elastic modulus is nonlinear. Therefore,

$$
\begin{aligned}
& S_{1}=S_{1}^{\text {lin }} ; \quad S_{3}=S_{3}^{\text {lin }} ; \quad S_{4}=S_{4}^{\text {lin }} ; \\
& S_{2}=S_{2}^{\text {lin }}\left[1+\beta_{2} \epsilon_{11}+\beta_{1} \epsilon_{22}+\delta_{2} \epsilon_{11}^{2}+\delta_{1} \epsilon_{22}^{2}\right] .
\end{aligned}
$$

- CASE B: in a more general case, we assume

$$
\begin{aligned}
& S_{1}=S_{1}^{\text {lin }}\left[1+\beta_{1} \epsilon_{11}+\beta_{2} \epsilon_{22}+\delta_{1} \epsilon_{11}^{2}+\delta_{2} \epsilon_{22}^{2}\right] \\
& S_{2}=S_{2}^{\text {lin }}\left[1+\beta_{2} \epsilon_{11}+\beta_{1} \epsilon_{22}+\delta_{2} \epsilon_{11}^{2}+\delta_{1} \epsilon_{22}^{2}\right] \\
& S_{3}=S_{3}^{\text {lin }}\left[1+\beta_{1} \epsilon_{11}+\beta_{2} \epsilon_{22}+\delta_{1} \epsilon_{11}^{2}+\delta_{2} \epsilon_{22}^{2}\right] ; \\
& S_{4}=S_{4}^{\text {lin }} .
\end{aligned}
$$

Any nonlinearity in the elastic coefficient $S_{4}$ does not add any additional information to the results reported in this paper, and thus, for simplicity of notations we have assumed $S_{4}$ to be linear. Introducing other components to the nonlinear moduli will render the theoretically expected link between DAET results and expected results more complex, but without influencing significantly the accuracy.

a. Case A: Nonlinear $\mathrm{S}_{2}$. This case is simple enough to allow us to better understand in a simplified case the link between DAET and quasistatic modulus. Simulations are performed using the equations described above. The TOF is calculated and $\tilde{S}_{2}$ is derived using Eq. (23). Finally, the obtained modulus is plotted as a function of $\epsilon_{11}$, as shown in Fig. 3, for three different choices of the nonlinear parameters. As expected, a quadratic dependence is found. The theoretical solution (i.e., the quasistatic modulus) given by Eq. (19) is also shown in Fig. 3 (solid line) and the agreement is excellent.

Furthermore, a SAET experiment has also been performed, and results are reported as empty symbols in Fig. 3. The SAET experiment was performed replacing the sinusoidal pump wave with a step-like function with varying amplitude, applied to both sides of the sample. The probe was propagating in the sample once equilibrium for the step-like function was achieved and then the amplitude of the pump was varied. In simulations, SAET experiments could be easily performed in both compression and tension. Results of the SAET experiment are in excellent agreement with those of a DAET calculation, as expected.

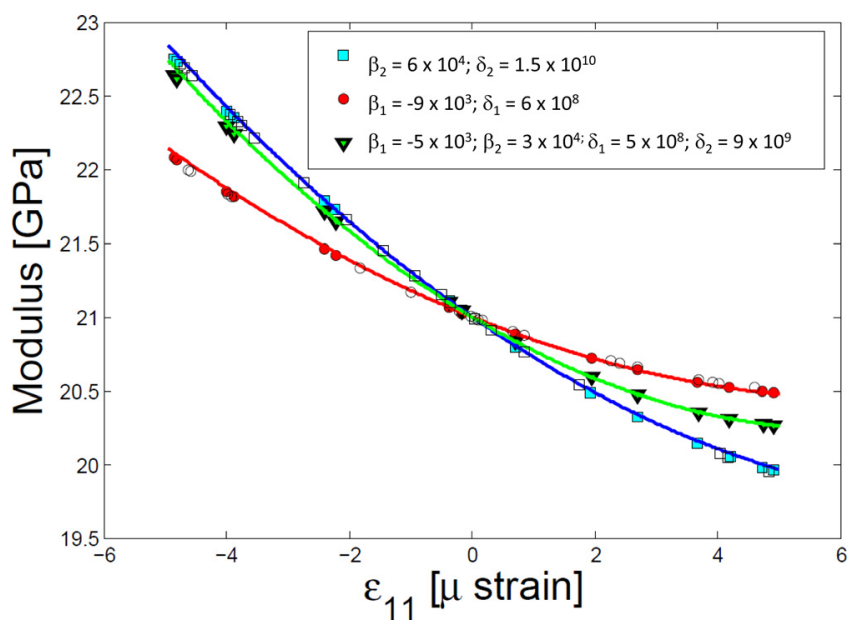

FIG. 3. Comparison between DAET measured modulus (solid symbols) and quasistatic modulus (solid line) in case A: $S_{2}$ is the only nonlinear modulus. Curves with empty symbols correspond to results obtained simulating a SAET experiment. 


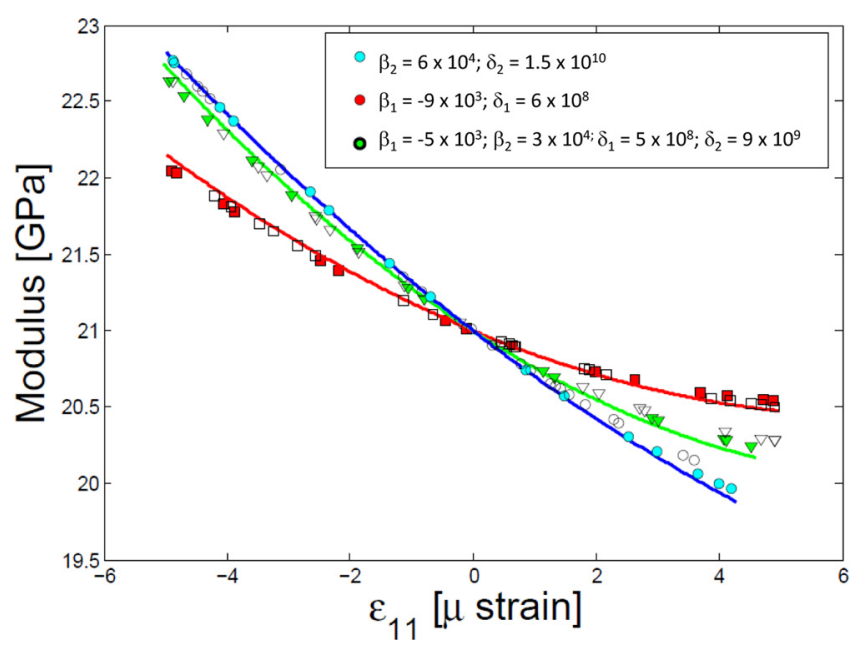

FIG. 4. Comparison between DAET measured modulus (solid symbols) and quasistatic modulus (solid lines) when $S_{1}, S_{2}$, and $S_{3}$ are nonlinear: case B. Curves with empty symbols correspond to results obtained simulating a SAET experiment.

A quantitative comparison of the nonlinear parameters estimated using DAET/SAET with the theoretical values (estimated from quasistatic measurements) demonstrates the validity of the approach. In fact, errors in the estimation of the parameters are less than $3 \%$ for both $\beta$ and $\delta$, thus well within the level of accuracy of a DAET laboratory experiment, which was estimated between $10 \%$ and $20 \%$.

We can conclude that the nonlinear parameters estimated using DAET agree well with the quasistatic predictions. However, no information about the individual components of the nonlinear modulus can be obtained, except in specific conditions. In fact, DAET parameters correspond to a proper combination of nonlinear parameters (Eq. (20)).

b. Case B: Nonlinear $\mathrm{S}_{1}, \mathrm{~S}_{2}$, and $\mathrm{S}_{3}$. Simulations are performed, $\tilde{S}_{2}$ is derived using Eq. (23), and it is plotted as a function of $\epsilon_{11}$, as shown in Fig. 4, for three different choices of the nonlinear parameters (same as those used in Fig. 3). As expected, a quadratic dependence is found again and agrees well, even though with less accuracy with respect to the previous case, with the theoretical solution given by Eq. (19), reported as a continuous line in Fig. 4. The agreement with results of SAET simulations (empty symbols) is again excellent.

A quantitative comparison of the nonlinear parameters estimated using DAET/SAET with the theoretical values (estimated from quasistatic measurements) indicates that errors are increasing up to $8 \%$, although still within the level of accuracy of a DAET laboratory experiment.

\section{Strong classical nonlinearity}

In Subsection III A, we have compared the results of DAET simulations with an approximate solution (Eq. (19)), which was derived considering the Poisson ratio to be strain independent, i.e., linear. This is of course not always the case. For the cases considered above, the difference between the exact (Eq. (17)) and approximate solutions was very small. However, when increasing the nonlinearity of the sample, the agreement becomes poorer.

In Fig. 5, the modulus estimated from DAET (symbols) is compared with the exact and approximate solutions: subplot (a) corresponds to case A3, while subplot (b) corresponds to
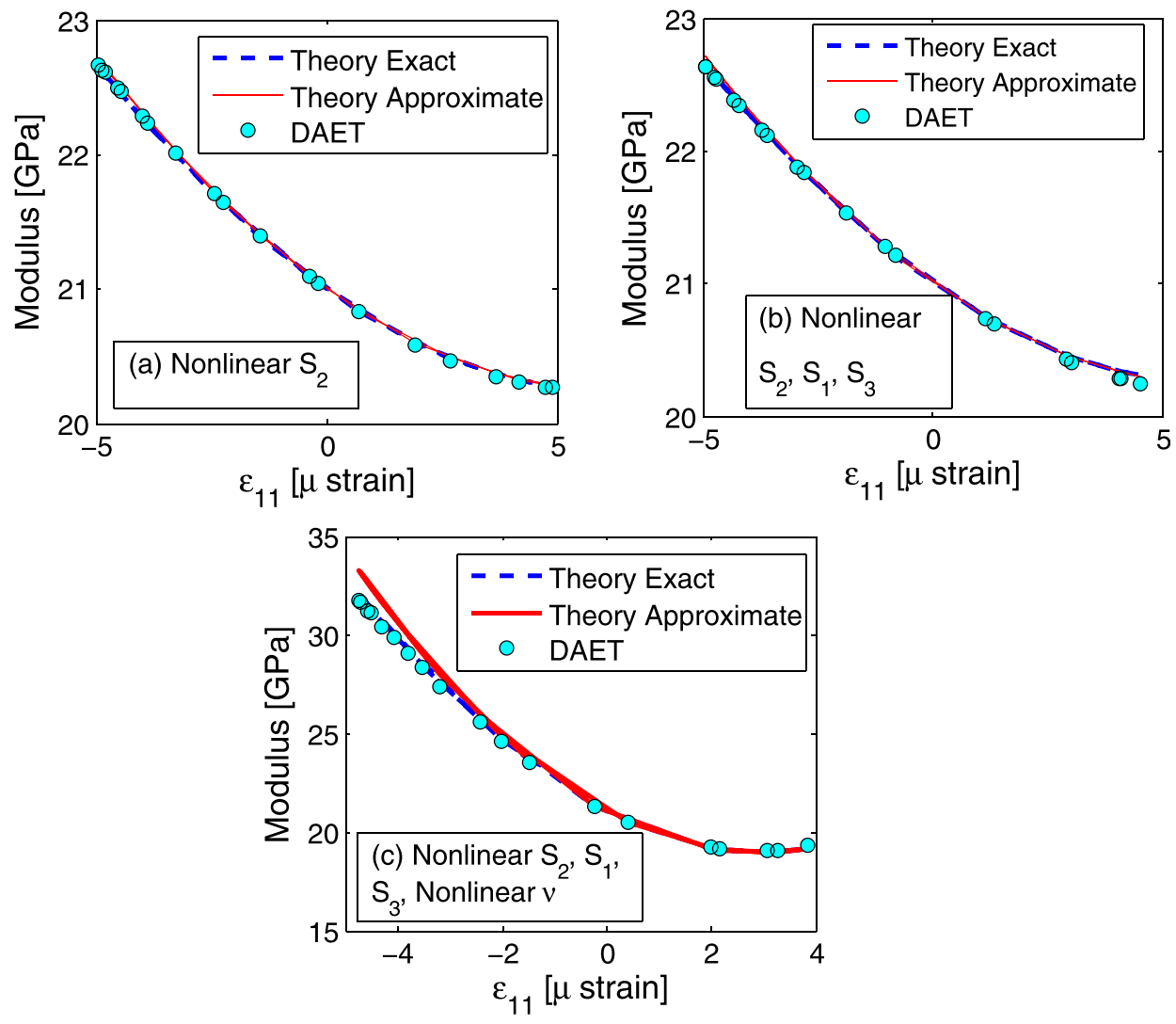

FIG. 5. Comparison between DAET measured modulus and theoretical predictions using the approximate (Eq. (19)) and the exact (Eq. (17)) solutions. (a) Only $S_{2}$ is nonlinear (case A3); (b) All moduli are nonlinear (case B3); (c) same as (b) but with a stronger nonlinearity $\left(\beta_{1 / 2}\right.$ and $\delta_{1 / 2}$ are multiplied by 5). 
case B3. As noticeable, for both we have an excellent agreement, slightly better with the exact solution. Indeed, in this case, the Poisson ratio was noticed to be almost completely independent from the strain.

This is no longer true in the case of a stronger nonlinearity in subplot (c). Here, both $\beta$ and $\delta$ have been multiplied by a factor 5. The Poisson ratio is highly nonlinear and thus the approximate solution is no longer valid. The agreement with the exact solution is still excellent, but a significant $a$ priori knowledge about the material properties is absolutely necessary to provide an interpretation to the parameters measured with DAET. Therefore, the knowledge/measurement of both components of the pump strain $\left(\epsilon_{11}\right.$ and $\left.\epsilon_{22}\right)$ is necessary to build the exact theoretical solution. Notice that in this case there is a significant deviation from a quadratic behavior.

In the case of classical nonlinearity, the nonlinear parameters are usually not huge, and thus the approximate solution can be considered as an excellent choice for most (or even all) experiments, as clearly shown by subplots (a) and (b) in Fig. 5. However, this could be not the case when nonclassical nonlinearity is present.

\section{Hysteretic nonlinearity}

The case of hysteretic nonlinearity provides two significant insights: the emergence of a loop in the modulus-strain relation and a strong contribution due to nonlinear attenuation. Again, simulations have been performed varying the nonlinearity of the sample, being in all cases $S_{2}$ the only nonlinear modulus. In particular, we have considered three cases: a weak and a strong dependence of $S_{2}$ on $\epsilon_{22}$ and a strong dependence of $S_{22}$ on both $\epsilon_{11}$ and $\epsilon_{22}$.

The results are reported in Fig. 6 where the modulus calculated from DAET simulations (red symbols), SAET simulations (cyan symbols), and from a quasistatic numerical experiment (solid line) are reported. For the quasistatic experiment, the modulus dependence on strain has been found implementing Eq. (14) and calculating the partial derivative of the stress vs. strain. The agreement between DAET and SAET is again very good, while poor agreement is found with theoretical results. As remarked, we ascribe the discrepancy to a significant nonlinearity of the attenuation coefficient. In both experiments ${ }^{29}$ and simulation results, ${ }^{33}$ it has been shown that the attenuation variation, as a function of strain, is of the same order of magnitude of the variation of the modulus and its hysteretic loop presents similarities with that of the modulus. Finally, we wish to observe that the discrepancy between DAET results and quasistatic expectations agrees well with experimental observations. Indeed, the quasistatic analysis highlights a discontinuity in the modulus dependence on strain in correspondence of the maximum/minimum of strain, which is never observed in a DAET experiment, similarly to what reported here.

The analysis of the curves reported in Fig. 6 is more complex than in the classical case. As already mentioned, one approach is to fit the upgoing and downgoing branches independently with a quadratic function, thus extracting two couple of parameters $\beta^{ \pm}$and $\delta^{ \pm}$. Another approach generally implemented to analyze experimental data is to fit the curve with a single quadratic function. ${ }^{22,29}$ The results of such a fit operated on DAET data is reported in Fig. 6 as a dashed red line. The agreement with the similar fit operated on quasistatic results (dashed blue line) is good.

\section{CONCLUSIONS}

We have discussed here the difficulties and problems related to the interpretation of the nonlinear parameters $\beta_{D}$ and $\delta_{D}$ measured in a DAET experiment. For this purpose, we have limited our analysis to numerical data, since they allow to have control of the expected solution (i.e., to estimate the quasistatic modulus) and to simulate in both compression and tension SAET experiments, which constitute a reliable solution to be compared with DAET.

This analysis has allowed us to show how DAET, if performed in proper experimental conditions, is equivalent to SAET, with the advantage of being faster to implement and of making it possible an analysis in both tension and compression. However, as expected, the techniques (both DAET and SAET) allow in general to estimate only a proper combination of the nonlinear elastic moduli. We have also shown that some difficulties might be present in the case of materials with a strong nonlinear attenuation and/or with a nonlinear dependence of the Poisson ratio. In these cases, the link of the DAET/SAET measured modulus with the quasistatic modulus could be more complex and difficult to predict.
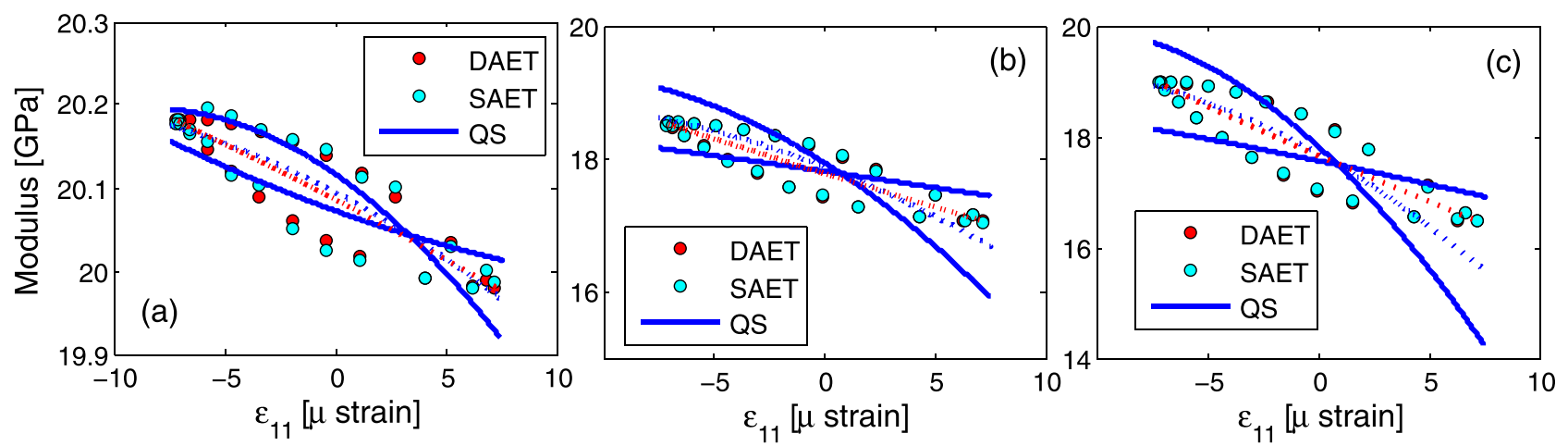

FIG. 6. Comparison between DAET measured modulus, SAET measured modulus, and quasistatic predictions (QS) for different choices of nonclassical nonlinear parameters. The dashed lines represent a fit of the DAET (red) and quasistatic (blue) results, obtained fitting at the same time the upgoing and downgoing branches of the corresponding curves. (a) $r_{1}=75 ; r_{2}=25$; (b) $r_{1}=50 ; r_{2}=50$; (c) $r_{1}=50 ; r_{2}=25 ; r_{3}=25$. Only the modulus $S_{2}$ is nonlinear and the parameters $r_{2}$ and $r_{3}$ characterize its dependence on $\epsilon_{11}$ and $\epsilon_{22}$, respectively (see Eq. (14) and following definitions). 
An additional issue to be considered for an accurate physical interpretation of the measured DAET parameters should also be noticed. In Eq. (15), it is assumed the pulse to be a longitudinal wave, which is not the case for anisotropic media, where Christoffel equations are defining the wave velocity as a combination of complex functions of the linear moduli. However, if the anisotropy is induced only by nonlinearity, given the symmetry of the pump strain, the propagation direction of the probe is along a principal direction, thus we are in the case of longitudinal wave propagation. Even though in our opinion this condition is satisfied in most cases, care must be taken when applying the DAET method to test strongly anisotropic media or with strongly localized and/or directional nonlinearities.

As mentioned, DAET should be implemented in specific experimental conditions. We have also analyzed the errors introduced when varying the experimental parameters: amplitude of the probe wave, alignment of transducers, and ratio between TOF and pump period. Results are reported in the supplementary material. In the case of an acceptable error up to $10 \%$, the experimental configurations could be considered quite robust: it is acceptable to use probe amplitudes less than $1 / 10$ of the pump amplitude, to align transducers with a precision of a few $\mathrm{mm}$ and to choose a pump period larger than about 10 times the TOF. Our results indicate that the experimental configuration usually adopted in the literature is sufficiently robust to allow the development of DAET based techniques for localized damage detection, localization, and monitoring. ${ }^{25,31}$

In our opinion, two main goals have still to be achieved to make DAET a complete technique. On one side, it is important to evaluate correctly the role of the attenuation coefficient in the definition of the velocity and to estimate the correction to be considered when attenuation is strongly strain dependent. Furthermore, the definition of a combination of DAET measurements properly coupled is needed in order to estimate the individual components of the nonlinear moduli of solid anisotropic media.

\section{ACKNOWLEDGMENTS}

The numerical simulations were performed with the support of HPC@POLITO CASPER3 node.

${ }^{1}$ B. Zinszner, P. A. Johnson, and P. N. J. Rasolofosaon, J. Geophys. Res. 102, 8105-8120, doi:10.1029/96JB03225 (1997).

${ }^{2}$ T. J. Ulrich and T. W. Darling, Geophys. Res. Lett. 28, 2293-2296, doi:10.1029/2000GL012480 (2001).

${ }^{3} \mathrm{H}$. Wang and M. Li, J. Appl. Phys. 113, 213515 (2013).

${ }^{4}$ R. A. Guyer and P. A. Johnson, Phys. Today 52, 30-35 (1999).

${ }^{5}$ P. A. Johnson, B. Zinszner, and P. N. J. Rasolofosaon, J. Geophys. Res. 101, 11553-11564, doi:10.1029/96JB00647 (1996).
${ }^{6}$ M. Scalerandi, M. Griffa, P. Antonaci, M. Wyrzykowski, and P. Lura, J. Appl. Phys. 113, 154902 (2013).

${ }^{7}$ C. Campos-Pozuelo, C. Vanhille, and J.-A. Gallego-Juarez, IEEE Trans. Ultrason., Ferroelectr. Freq. Control 53, 175-184 (2006).

${ }^{8}$ M. Scalerandi, A. S. Gliozzi, and D. Olivero, J. Nondestr. Eval. 33, 269-278 (2014).

${ }^{9}$ S. Haupert, S. Guerard, F. Peyrin et al., PLoS One 9, e83599 (2014).

${ }^{10}$ F. Ciampa et al., J. Nondestr. Eval. 33, 515-521 (2014).

${ }^{11}$ M. Boudjema, I. B. Santos, K. R. McCall et al., Nonlinear Process. Geophys. 10, 589-597 (2003).

${ }^{12}$ M. Scalerandi, A. S. Gliozzi, C. L. E. Bruno, and P. Antonaci, Phys. Rev. B 81, 104114 (2010).

${ }^{13}$ J. A. TenCate, E. Smith, and R. A. Guyer, Phys. Rev. Lett. 85, 1020-1023 (2000).

${ }^{14}$ P. A. Johnson and A. Sutin, J. Acoust. Soc. Am. 117, 124-130 (2005).

${ }^{15}$ G. Renaud, S. Calle, and M. Defontaine, Appl. Phys. Lett. 94, 011905 (2009).

${ }^{16} \mathrm{~J}$. Jaeger and N. Cook, Fundamentals of Rock Mechanics, 3rd ed. (Chapman and Hall, London, 1979), p. 78.

${ }^{17}$ D. S. Hughes and J. L. Kelly, Phys. Rev. 92, 1145 (1953).

${ }^{18}$ K. W. Winkler and L. McGowan, J. Geophys. Res. 109, B10204, doi:10.1029/2004JB003262 (2004).

${ }^{19}$ M. Destrade, M. D. Gilchrist, and R. W. Ogden, J. Acoust. Soc. Am. 127, 2103-2106 (2010).

${ }^{20}$ I. Lillamand, J.-F. Chaix, M.-A. Ploix, and V. Garnier, NDT \& E Int. 43, 655-660 (2010).

${ }^{21}$ J.-L. Gennisson, M. Renier, S. Catheline et al., J. Acoust. Soc. Am. 122, 3211-3219 (2007).

${ }^{22}$ J. Rivière, G. Renaud, R. A. Guyer, and P. A. Johnson, J. Appl. Phys. 114, 054905 (2013).

${ }^{23}$ G. Renaud, P.-Y. Le Bas, and P. A. Johnson, J. Geophys. Res. 117, B06202, doi:10.1029/2011JB009127 (2012).

${ }^{24}$ F. Moradi-Marani, S. A. Kodjo, P. Rivard et al., J. Nondestr. Eval. 33, 288-298 (2014)

${ }^{25}$ J. Rivière, M. C. Remillieux, Y. Ohara, B. E. Anderson, S. Haupert, T. J. Ulrich, and P. A. Johnson, J. Nondestr. Eval. 33, 216-225 (2014).

${ }^{26}$ D. Bui, S. A. Kodjo, P. Rivard et al., J. Nondestr. Eval. 32, 25-36 (2013).

${ }^{27}$ T. Gallot, A. Malcolm, T. L. Szabo et al., J. Appl. Phys. 117, 034902 (2015).

${ }^{28}$ S. Haupert, G. Renaud, J. Riviere et al., J. Acoust. Soc. Am. 130, 2654-2661 (2011).

${ }^{29}$ G. Renaud, J. Riviere, S. Haupert et al., J. Acoust. Soc. Am. 133, 3706-3718 (2013).

${ }^{30}$ G. Renaud, S. Calle, J.-P. Remenieras et al., IEEE Trans. Ultrason., Ferroelectr. Freq. Control 55, 1497-1507 (2008).

${ }^{31}$ S. Haupert, J. Riviere, B. E. Anderson et al., J. Nondestr. Eval. 33, 226-248 (2014).

${ }^{32}$ See supplementary material at http://dx.doi.org/10.1063/1.4931917 for the analysis of the influence of the experimental set-up parameters on reliability of a DAET measurement.

${ }^{33}$ A. S. Gliozzi and M. Scalerandi, J. Acoust. Soc. Am. 136, 1530-1541 (2014).

${ }^{34}$ P. P. Delsanto and M. Scalerandi, J. Acoust. Soc. Am. 104, 2584-2591 (1998).

${ }^{35}$ B. Capogrosso Sansone and R. A. Guyer, Phys. Rev. B 66, 224101 (2002).

${ }^{36}$ P. P. Delsanto and M. Scalerandi, Phys. Rev. B 68, 064107 (2003).

${ }^{37}$ O. Bou Matar, P.-Y. Guerder, Y. F. Li et al., J. Acoust. Soc. Am. 131, 3650-3663 (2012).

${ }^{38}$ M. Scalerandi, S. Idjmarene, M. Bentahar, and R. El Guerjouma, Commun. Nonlinear Sci. Numer. Simul. 22, 334-347 (2015).

${ }^{39}$ Y. F. Li, O. Bou Matar, B. S. Li et al., Wave Motion 52, 54-65 (2015).

${ }^{40}$ J. Qiao, A. V. Amirkhizib, K. Schaaf, S. Nemat-Nasserb, and G. Wu, Mech. Mater. 43, 598-607 (2011). 